\title{
Immune gene tied to social behavior in autism mouse model
}

\author{
BY LAURA DATTARO
}

3 FEBRUARY 2022

\section{Listen to this story:}

https://www.spectrumnews.org/wp-content/uploads/2022/02/audio-f0f0f2cb-6a60-469db39a-13efadf61f53-encodings.mp3

Activating the immune system blunts social behaviors in mice missing a copy of the autism-linked gene SHANK3, according to a new study. But blocking TRPV4, a gene that encodes an ion channel involved in inflammatory responses, can reverse the change, the researchers also found.

The results may clarify why autism traits can vary widely among people with mutations in SHANK3 - and why mice missing a copy of the gene don't consistently show autism-like traits, says lead investigator Camilla Bellone, associate professor of basic neurosciences at the University of Geneva in Switzerland.

"Genetics by itself cannot explain the heterogeneity," Bellone says. "There has to be something else in place."

Bellone and her colleagues found that when they reduced SHANK3 expression in the brains of wildtype mice after birth, it also altered the expression of genes involved in the body's inflammatory response. This observation led them to suspect that a strong immune response say, in response to severe infection - could induce behavioral changes in SHANK3 mice.

Having such an infection during pregnancy increases a woman's chances of having a child with autism or autism-like traits. Prompting an immune response in pregnant mice similarly ups the chances of autism-like traits in their pups.

"This paper is a beautiful piece of this puzzle trying to figure out how this immune activation actually has an important impact in neurodevelopment," says Manuel López Aranda, assistant 


\section{Spectrum | Autism Research News}

https://www.spectrumnews.org

project scientist in Alcino Silva's lab at the University of California, Los Angeles, who was not involved in the work.

The researchers injected the nucleus accumbens - a brain region involved in social behavior - of wildtype mice with a viral vector carrying genetic material to lower the expression of SHANK3. Mice injected six days after birth, but not 90 days after, showed unusually low interest in other mice as adults, even though both had similarly low levels of SHANK3 expression in the brain.

The lowered SHANK3 expression, in turn, altered the expression of another 178 genes, many of which are involved in the immune system - a finding Bellone calls "striking." One gene, TRPV4, was upregulated in the mice injected early but not in controls, suggesting a potential link with the animals' social changes.

Infusing a drug that inhibits TRPV4 expression into the nucleus accumbens of these mice boosted their sociability.

The researchers then tested sociability in mice missing one copy of SHANK3 and found no differences from wildtype mice. But when researchers injected them with a bacterial molecule to stimulate their immune systems 24 hours before the behavior test, the SHANK3 mice became less social, whereas the wildtype mice did not. The social changes disappeared within one week of injection.

Inhibiting TRPV4 in SHANK3 mice exposed to immune activation improved their social behavior, the researchers found.

The findings were published in Molecular Psychiatry in January.

Although many studies probe immune activation in utero, when autism is thought to emerge, doing so after birth in mice can also provide useful information, says Devanand Manoli, assistant professor of psychiatry at the University of California, San Francisco, who was not involved the work. Brain development six days after birth in the rodents is similar to that during late gestation in people.

"From a general neural development sensitization standpoint, there's an analogy that's a valid one," Manoli says. "That's a pretty reasonable claim to make."

The findings don't necessarily point to a cause of autism in people with SHANK3 mutations, he says, but rather demonstrate that such mutations can make the brain more vulnerable to 'second hits,' such as immune activation.

"It's the interaction that's important," he says. 


\section{Spectrum | Autism Research News}

https://www.spectrumnews.org

The work also reveals an important time period during which the brain might be most susceptible to immune activation, López Aranda says.

"It's not only the gene mutation, it's not only the immune activation, it's the combination of both," he says. "And it's the combination of both in a specific window of development."

Provoking an immune response in mouse pups missing a copy of TSC2, another gene linked to autism, leads to social memory problems when the male mice hit adulthood, López Aranda previously showed. And autistic boys are more likely than non-autistic boys to have had a serious infection in early childhood, he found.

The researchers next plan to study the effects of immune activation on SHANK3 mice earlier in development, Bellone says, and to repeat the experiments in mice with mutations in other autismlinked genes.

\section{Cite this article: https://doi.org/10.53053/BMSO3887}

